\title{
Simulation of the magnetic system of a linear motor for a delimber
}

\author{
Sergey Antonov, Gennady Nikitenko, Andrey Adoshev, Igor Devederkin, Alexey Efanov
}

Stavropol State Agrarian University, Russia

\begin{abstract}
The existing pruning shears and delimbers have many drawbacks that limit their widespread use in the production process. These are such disadvantages as large weight and dimensions, high power consumption, vibration and noise, low mobility due to being tied to an energy source. DC motors are used to drive the cutting blades. Their main disadvantage is the low operational reliability of such an element as electric brushes. The use of the kinematic transformation of the rotational motion of the electric motor into the reciprocating motion of the blades reduces the overall efficiency of the device and increases the consumption of electrical energy. The proposed linear electric motor for the delimber drive will increase the efficiency, operational reliability and reduce energy consumption for cutting tree branches. A feature of a linear electric motor is the use of two magnetizing coils, which are switched on alternately. The use of a thin element in the magnetic system makes it possible to redistribute the obtained magnetic flux towards the armature of the linear electric motor. The armature of the electric motor consists of magnetic and nonmagnetic bushings of a certain design. This allows you to obtain a magnetic flux, which, passing along the armature, creates an electromagnetic force that sets it in motion. The cutting blade is then anchored. This allows you to improve the characteristics of the proposed delimber. The main challenge in the design of the delimber is to create the maximum force on the cutting blade required to cut branches. For this, it is necessary to perform an improvement of the magnetic system of the linear electric motor. For this purpose, the simulation of the magnetic system was carried out in the ElCut program.
\end{abstract}

\section{Introduction}

Modern agriculture is aimed at obtaining maximum yields. In practice, to increase yields, various agrotechnical measures are used. One of the agrotechnical measures that increase yields in industrial horticulture is detailed pruning of tree branches. Secateurs are used to cut branches with a diameter of up to $15 \mathrm{~mm}$. Branches with a diameter of more than $30 \mathrm{~mm}$ are removed with a hacksaw. Branches with a diameter of 15 to $30 \mathrm{~mm}$ are cut with more powerful secateurs or delimbers. According to the results of studies carried out in the gardens of the Kabardino-Balkarian Republic, these branches are about $30 \%$ of the total number of cuttings $[1 ; 2 ; 3 ; 4]$.

Modern domestic industry does not produce serial mechanized and electrified tools for pruning trees, for industrial horticultural enterprises; therefore, mainly manual delimbers are used [5-15]. The branches are removed by cutters, which are placed on a mobile platform (Fig. 1).

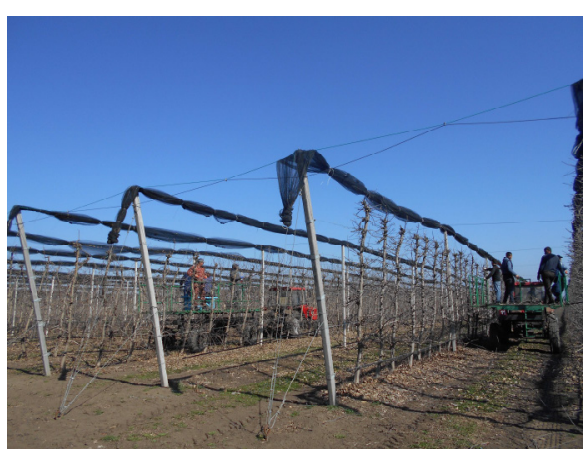

Fig. 1. Tree branches pruning

Manual delimbers are produced by the world industry RACO, 1553 ЦИ, Felco F220, Wirpo 07431 (Fig. 2.).

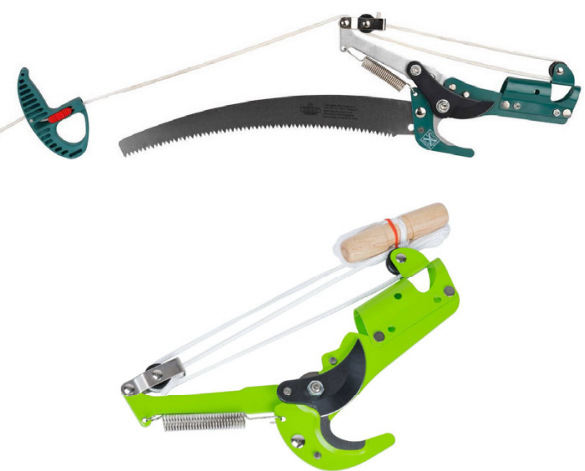

\footnotetext{
*Corresponding author: antonov_serg@mail.ru
} 

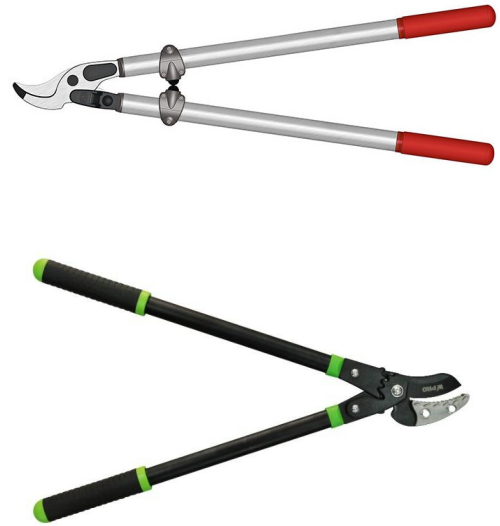

Fig. 2. Manual delimbers

Working with hand tools is ineffective, leading to a decrease in productivity and quality of the pruning process. There are many commercially available electric tools, such as the KARCHER lopper (Fig. 3).

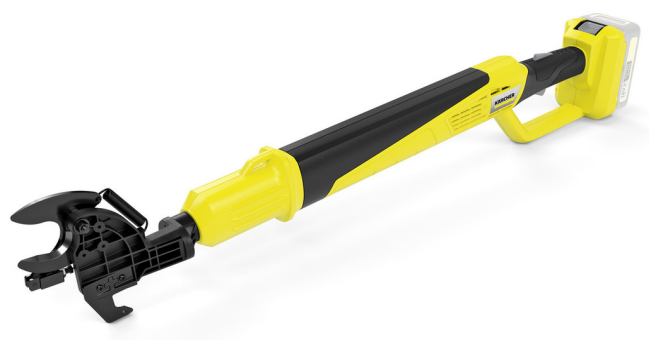

Fig. 3. Electric lopper KARCHER

The most promising instrument is the electrified instrument. An electrified tool has several advantages over others. These advantages are cut cleanliness, productivity, low energy consumption and mobility. Existing electric loppers use DC motors to drive the cutting pair. Using a DC motor will shorten the life of the tool. This is due to the presence of electric brushes, which have low reliability. To protect the electric motor from environmental influences, it is necessary to increase the degree of protection (IP) against water and dust entering the motor. This entails an increase in the cost of the tool and its weight. It should also be noted that to convert the rotational motion of the armature into the reciprocating motion of the blade, a kinematic transformation scheme is used. The element of the kinematic transformation increases the mass, reduces the efficiency and energy efficiency of the tool. As a replacement for this motor, we propose the use of a linear electric motor. It has a number of advantages, such as high energy efficiency, small weight and dimensions, and low noise level.

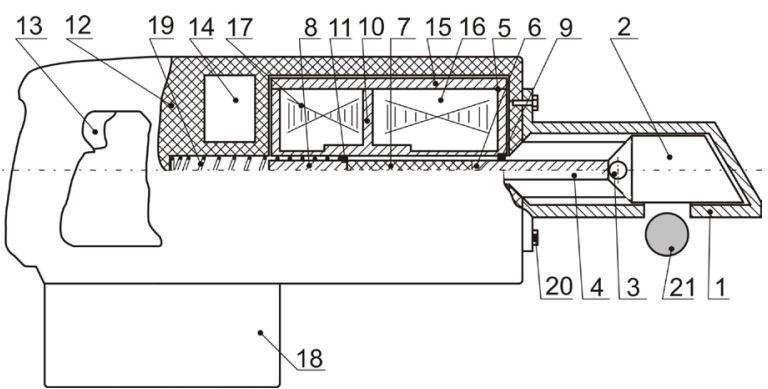

Fig. 4. The delimber

The delimber (Fig. 4.) consists of a fixed blade 1 in which a movable blade (2) is placed, connected with a screw (3) to the armature (4) of the linear electric motor (5). Armature (4) consists of an upper magnetic sleeve (6), a non-magnetic sleeve (7) and a lower magnetic sleeve (8). Armature (4) is installed using a nonmagnetic sliding bearing (9) in the magnetic circuit (10) and a non-magnetic sliding bearing (11) mounted on the lower magnetic sleeve (8). In the housing (12) are a power button (13), a control unit (14), a magnetic housing (15), the first magnetizing coil (16), the second magnetizing coil (17), the battery (18) and the spring (19). At the same time, the fixed blade (1) is secured to the housing (12) by means of a bolt (20). Tree branch (21) is located next to the delimbers (Fig. 4.).
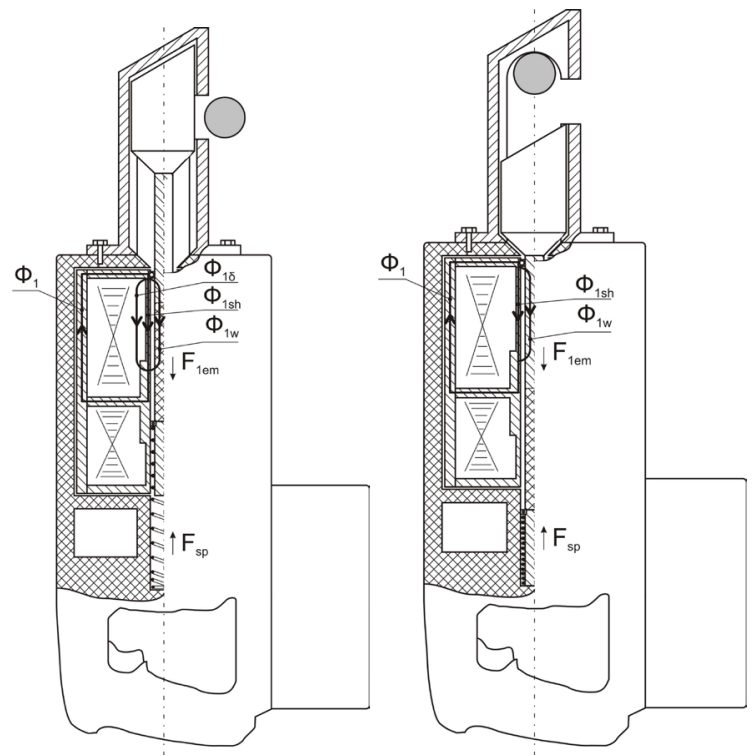

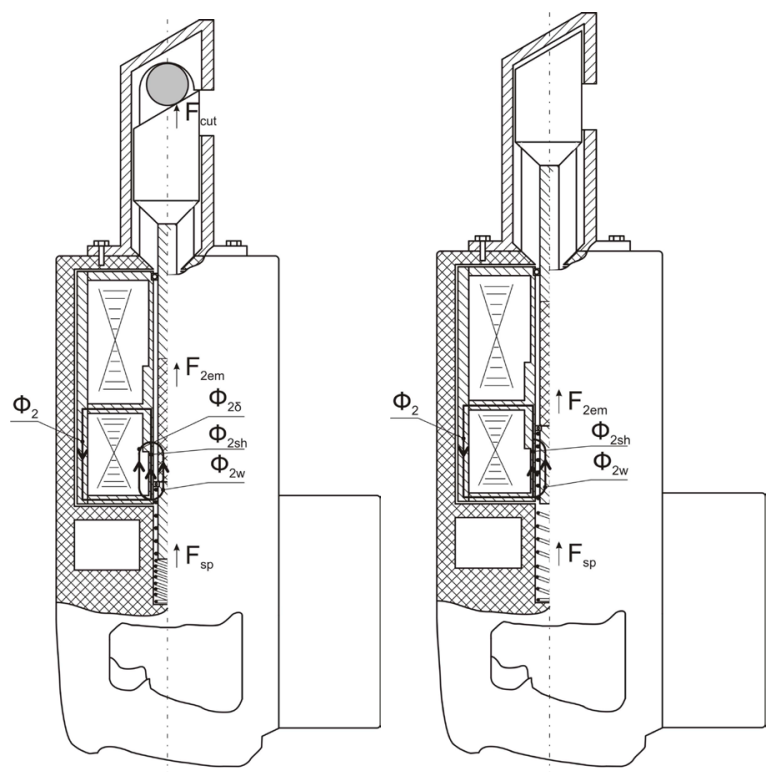

Fig. 5. Delimber operating principle

The principle of operation of the presented pruner (Fig. 5) is described in detail in article [16].

\section{Materials and methods}

The offered delimber is designed for cutting branches over $15 \mathrm{~mm}$. The linear motor of the lopper contains the armature (1), consisting of the first magnetic sleeve (2), non-magnetic sleeve (3), the second magnetic sleeve (4). Armature (1) is installed using sliding bearing (5) in the magnetic circuit (6). The first (7) and the second (8) magnetizing coils are located in the magnetic circuit (6), which are protected against the influence of the environment by the magnetic housing (9) (Fig. 6) .

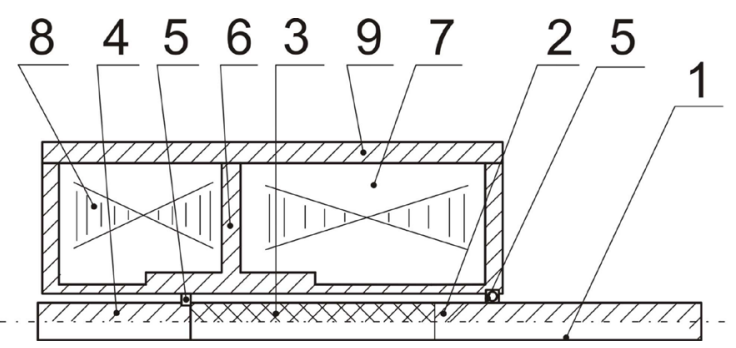

Fig. 6. Linear motor

The operation principle of a linear electric motor is based on the use of magnetic saturation of the magnetic circuit section, which leads to the pushing of the magnetic flux towards the armature [17]. In this case, an electromagnetic force begins to act on the armature, which leads to its movement. The magnitude of the electromagnetic force will depend on the geometric parameters of the magnetic system. The use of this magnetic field property requires the use of modern calculation methods. Since modeling by the method of circuits and the method of similarity theory give a low calculation accuracy, it is necessary to use the method of finite differences, or the method of finite elements. They provide an objective picture of the magnetic fields of a linear motor. The calculation of the finite element method is based on the Poisson equation:

$$
\begin{aligned}
& \frac{\partial}{\partial r}\left(v \frac{1}{r} \frac{\partial}{\partial r}(r A)\right)+\frac{\partial}{\partial z}\left(v \frac{1}{r} \frac{\partial}{\partial z}(r A)\right)=J \text { (1) } \\
& \text { where } v=\frac{1}{\mu_{0} \mu} \text { is a specific magnetic resistance; }
\end{aligned}
$$

$\mu_{0}$ is a magnetic permeability of vacuum;

$\mu$ is a magnetic permeability of matter;

$\mathrm{r}, \mathrm{z}$ are cylindrical coordinates.

The main task of modeling is to determine the most rational design of the magnetic system. The design efficiency will depend on the geometric parameters of the magnetic circuit.

One of these parameters is the length of the magnetic circuit thin section of the first and second magnetizing coils (Fig. 7).

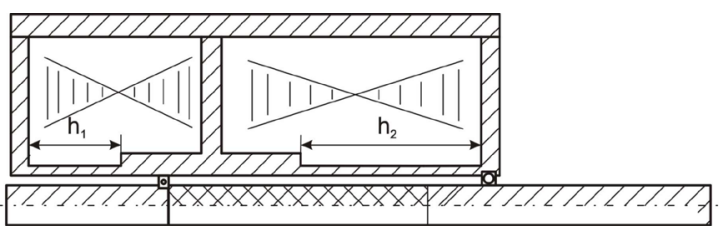

Fig. 7. Thin sections h1 and h2 of a linear motor

When designing and researching electrical machines, the calculation of the magnetic field is used. When calculating magnetic fields, the most important quantities are magnetic field strength, inductance, magnetic induction, magnetic moment and force. Modeling of the magnetic system of a linear electric motor was carried out in the ElCut software application. The used program allows solving linear and nonlinear problems of magnetostatics. At the first stage, you need to set the geometric parameters of the electric motor. Next, the parameters of the magnetic system, the magnetizing coil, and the boundary conditions (Dirichlet condition, Neumann condition) are set. Then a finite element mesh is applied to the geometric model. The smaller the grid step, the more accurate the solution can be obtained, but the calculation time increases.

\section{Results and discussion}

ElCut software allows you to calculate the magnetic system of a linear electric motor using the finite element method. The result of the calculation is the magnetic fluxes plot over structural elements (Fig. 8). The picture of the field can be represented as a color map, in the form of lines of force, in the form of a family of vectors. The color map is associated with a color scale, which indicates the corresponding value. Field lines are lines of equal potential or magnetic flux lines. Vectors are a family of directional line segments that carry information about a magnitude and its spatial direction. 


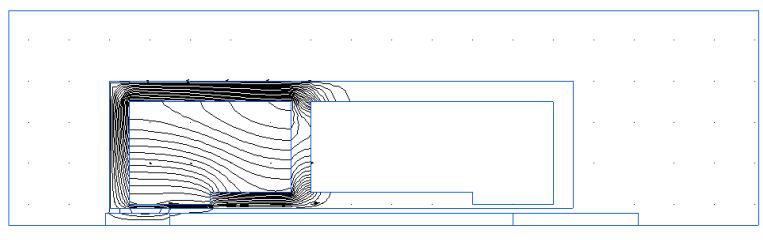

a

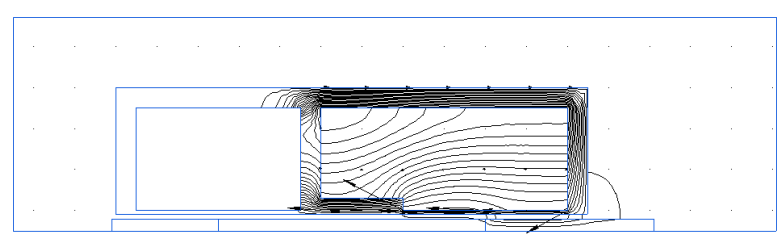

b

Fig. 8. The calculating result of the magnetic system of the delimber linear electric motor: a - the first magnetizing coil; $b$ - the second magnetizing coil

In the modeling course the values of the magnetic induction in the working gap were calculated for the first and second magnetizing coils, as a function of the length of the magnetic circuit thin section. The results of calculating the magnetic induction are presented in tables 1, 2 .

Table 1. Magnetic induction in the working gap of the first magnetizing coil $(\mathrm{T})$

\begin{tabular}{|c|c|c|c|c|}
\hline \multirow[t]{2}{*}{$\begin{array}{c}\text { Coil } \\
\text { length }\end{array}$} & \multicolumn{4}{|c|}{$\begin{array}{l}\text { Length of the thin section of the magnetic circuit }\left(h_{1}\right) \text {, } \\
\text { mm }\end{array}$} \\
\hline & 10 & 20 & 30 & 40 \\
\hline 0 & 0.25 & 0.3 & 0.27 & 0.25 \\
\hline 2 & 0.65 & 0.55 & 0.55 & 0.54 \\
\hline 4 & 0.67 & 0.56 & 0.55 & 0.54 \\
\hline 6 & 0.68 & 0.5 & 0.52 & 0.5 \\
\hline 8 & 0.45 & 0.31 & 0.32 & 0.28 \\
\hline 10 & 0.36 & 0.25 & 0.25 & 0.22 \\
\hline 12 & 0.4 & 0.3 & 0.23 & 0.2 \\
\hline 14 & 0.7 & 0.54 & 0.23 & 0.2 \\
\hline 16 & 1.4 & 1.05 & 0.26 & 0.18 \\
\hline 18 & 0.5 & 0.6 & 0.38 & 0.18 \\
\hline 20 & 0.22 & 0.55 & 0.7 & 0.22 \\
\hline 22 & 0.2 & 0.54 & 1.0 & 0.3 \\
\hline 24 & 0.2 & 0.52 & 0.47 & 0.5 \\
\hline 26 & 0.19 & 0.43 & 0.45 & 0.87 \\
\hline 28 & 0.19 & 0.18 & 0.44 & 0.45 \\
\hline 30 & 0.19 & 0.17 & 0.43 & 0.38 \\
\hline 32 & 0.18 & 0.17 & 0.42 & 0.37 \\
\hline 34 & 0.18 & 0.15 & 0.42 & 0.36 \\
\hline 36 & 0.18 & 0.15 & 0.4 & 0.35 \\
\hline 38 & 0.18 & 0.15 & 0.15 & 0.34 \\
\hline 40 & 0.18 & 0.15 & 0.12 & 0.34 \\
\hline
\end{tabular}

The magnitude of the magnetic induction in the working gap is the main parameter of the magnetic system. It affects the magnitude of the thrust force of the armature of the linear electric motor. Traction force ultimately affects the performance of a handheld electrified tool. This efficiency lies in the minimum consumption of electrical energy for the process of cutting tree branches.
Table 2. Magnetic induction in the working gap of the second magnetizing coil (T)

\begin{tabular}{|c|c|c|c|c|c|c|}
\hline \multirow{2}{*}{$\begin{array}{c}\text { Coil } \\
\text { length }\end{array}$} & \multicolumn{7}{|c}{ Length of the thin section of the magnetic circuit $\left(\mathbf{h}_{\mathbf{1}}\right)$} \\
\cline { 2 - 7 } & $\mathbf{1 0}$ & $\mathbf{2 0}$ & $\mathbf{3 0}$ & $\mathbf{4 0}$ & $\mathbf{5 0}$ & $\mathbf{6 0}$ \\
\hline 0 & 0.1 & 0.32 & 0.32 & 0.32 & 0.32 & 0.32 \\
\hline 2 & 0.12 & 0.4 & 0.4 & 0.39 & 0.4 & 0.39 \\
\hline 4 & 0.1 & 0.45 & 0.47 & 0.47 & 0.49 & 0.47 \\
\hline 6 & 0.5 & 0.35 & 0.39 & 0.37 & 0.4 & 0.37 \\
\hline 8 & 0.52 & 0.27 & 0.31 & 0.3 & 0.32 & 0.26 \\
\hline 10 & 0.55 & 0.29 & 0.3 & 0.27 & 0.3 & 0.25 \\
\hline 12 & 0.56 & 0.4 & 0.3 & 0.26 & 0.3 & 0.25 \\
\hline 14 & 0.53 & 0.8 & 0.31 & 0.26 & 0.3 & 0.25 \\
\hline 16 & 0.3 & 1.1 & 0.37 & 0.26 & 0.29 & 0.25 \\
\hline 18 & 0.7 & 0.57 & 0.6 & 0.26 & 0.29 & 0.25 \\
\hline 20 & 0.15 & 0.55 & 1.1 & 0.31 & 0.28 & 0.25 \\
\hline 22 & 0.17 & 0.55 & 0.57 & 0.4 & 0.28 & 0.25 \\
\hline 24 & 0.18 & 0.53 & 0.5 & 0.78 & 0.28 & 0.23 \\
\hline 26 & 0.19 & 0.3 & 0.9 & 1.05 & 0.3 & 0.22 \\
\hline 28 & 0.2 & 0.19 & 0.49 & 0.48 & 0.4 & 0.25 \\
\hline 30 & 0.21 & 0.19 & 0.5 & 0.45 & 0.8 & 0.29 \\
\hline 32 & 0.22 & 0.19 & 0.5 & 0.43 & 1.08 & 0.4 \\
\hline 34 & 0.23 & 0.19 & 0.49 & 0.43 & 0.5 & 0.7 \\
\hline 36 & 0.24 & 0.19 & 0.3 & 0.43 & 0.42 & 0.95 \\
\hline 38 & 0.24 & 0.19 & 0.15 & 0.43 & 0.4 & 0.5 \\
\hline 40 & 0.24 & 0.18 & 0.15 & 0.43 & 0.4 & 0.4 \\
\hline 42 & 0.25 & 0.18 & 0.15 & 0.43 & 0.4 & 0.36 \\
\hline 44 & 0.25 & 0.18 & 0.15 & 0.43 & 0.4 & 0.35 \\
\hline 46 & 0.25 & 0.18 & 0.15 & 0.3 & 0.4 & 0.35 \\
\hline 48 & 0.25 & 0.18 & 0.15 & 0.15 & 0.4 & 0.34 \\
\hline 50 & 0.25 & 0.17 & 0.15 & 0.13 & 0.4 & 0.34 \\
\hline 52 & 0.25 & 0.17 & 0.14 & 0.12 & 0.4 & 0.33 \\
\hline 54 & 0.25 & 0.17 & 0.14 & 0.12 & 0.4 & 0.33 \\
\hline 56 & 0.25 & 0.17 & 0.14 & 0.12 & 0.25 & 0.32 \\
\hline 58 & 0.25 & 0.17 & 0.14 & 0.12 & 0.08 & 0.32 \\
\hline 60 & 0.25 & 0.17 & 0.14 & 0.12 & 0.08 & 0.32 \\
\hline
\end{tabular}

Based on the data given in the tables, we can say that when the first magnetizing coil operates at different lengths of the thin section, the maximum value of the magnetic induction $(1.4 \mathrm{~T})$ in the working gap decreases with increasing this length. For the second magnetizing coil, the maximum value of the magnetic induction (1.1 $\mathrm{T})$ practically does not change. This gives reason to say that such a parameter as the length of a thin section of the magnetic circuit does not always affect the effective redistribution of magnetic fluxes towards the armature. Increasing the efficiency of this redistribution in further studies should be considered in the direction of changing the geometric parameters of the armature elements. In addition, it is necessary to carry out calculations for short-term, intermittent and long-term operating modes of the electric motor.

\section{Conclusions}

After analyzing the obtained results, we can say that the maximum value of the magnetic induction for the first magnetizing coil with a thin section length of $10 \mathrm{~mm}$ will be equal to $1.4 \mathrm{~T}$ at a distance of $16 \mathrm{~mm}$ from the beginning. The maximum value of the magnetic induction for the second magnetizing coil with a thin section length of 20 and $30 \mathrm{~mm}$ will be equal to $1.1 \mathrm{~T}$ at a distance of 16 and $20 \mathrm{~mm}$, respectively. 


\section{References}

1. U.P. Hedrick Cyclopedia of Hardy Fruits. (New York: The MacMillan Company, 1922)

2. E. Csanády, E. Magoss Mechanics of Wood Machining. 2-nd ed. (Springer-Verlag Berlin Heidelberg, 2013)

3. P.M. Tavasiev Means of small-scale mechanization for fruit plantations of peasant (farmer) farms): $\mathrm{PhD}$ diss. (Vladikavkaz, 2009).

4. O.I. Turiev Development and justification of the main parameters of the cutter branches for peasant (farm) farms): ( $\mathrm{PhD}$ diss. Vladikavkaz, 1999).

5. J. Wang, G.W. Jewell, and D. Howe, IEEE Trans., 35(2), 1986-2000, (1999),

6. S. Chapman Electric Machinery Fundamentals. (USA: McGraw-Hill Science, 2005).

7. D. V. Hoang, D. T. Trong, Z. Ivan, K. S. Bong, P.T. Thanh (eds.) AETA 2017 - Recent Advances in Electrical Engineering and Related Sciences: Theory and Application.(Springer, 2018)

8. J. Wang and D. Howe, IEEE Trans., 41(9), 24702489 (2005),

9. D.C.J. Krop, E.A Lomonova, A.J.A. Vandenput, IEEE Trans., 44(3), 352-359, (2008)

10. Y.-W. Zhu and Y.-H. Cho, IEEE Trans., 43(6), , 2537-2539 (2007)

11. P. Zheng, A. Chen, P. Thelin, W.M. Arshad, and Ch. Sadarangani, IEEE Trans., 43(1), , 447-449, (2007)

12. J. Prudell, M. Stoddard, E. Amon, T.K.A. Brekken, and A. von Jouanne, IEEE Trans.,46(6), 2392-2400, (2010)

13. S. Niu, S.L. Ho, and W.N. Fu, IEEE Trans., 47(10), 3598-3601, (2011)

14. R.C. Holehous, K. Atallah, and J. Wang, IEEE Trans., 47(10), 4171-4174, (2011)

15. J. Wang, K. Atalah, and W. Wang, IEEE Trans., 47(10), 4477-4480, (2011).

16. S. Antonov, G. Nikitenko Engineering for rural development, (2020).

17. S. Antonov, G. Nikitenko, Proceeding of 7 th International Conference on «Trends in Agricultural Engineering 2019», Prague, Czech Republic, 2019, pp. 40-44 (2019) 